\title{
Revisiting the pion's scalar form factor in chiral perturbation theory
}

\author{
Andreas Jüttner \\ CERN, Physics Department, TH Unit, \\ CH-1211 Geneva 23, Switzerland \\ E-mail: juettner@mail.cern.ch
}

\begin{abstract}
The quark-connected and the quark-disconnected Wick contractions contributing to the pion's scalar form factor are computed in the two and in the three flavour chiral effective theory at next-to-leading order. While the quark-disconnected contribution to the form factor itself turns out to be power-counting suppressed its contribution to the scalar radius is of the same order of magnitude as the one of the quark-connected contribution. This result underlines that neglecting quark-disconnected contributions in simulations of lattice QCD can cause significant systematic effects. The technique used to derive these predictions can be applied to a large class of observables relevant for QCD-phenomenology.
\end{abstract}

KEYwORDS: Lattice QCD, Chiral Lagrangians

ARXIV EPRINT: 1110.4859 


\section{Contents}

1 Introduction 1

2 Quark disconnected diagrams in chiral effective theory 2

$3 \quad \mathrm{PQ} \chi \mathrm{PT}$ for the scalar pion form factor $\quad 4$

4 Results for the form factor $\quad 5$

4.1 Expressions in the effective theory at NLO 5

$\begin{array}{lll}4.2 & \text { Numerical results } & 6\end{array}$

$\begin{array}{lll}5 & \text { Applications } & 7\end{array}$

$\begin{array}{llr}6 & \text { Conclusions and outlook } & 9\end{array}$

$\begin{array}{ll}\text { A Vertices in the partially quenched theory } & 10\end{array}$

$\begin{array}{lll}\text { A.1 Vertices } & 10\end{array}$

$\begin{array}{lll}\text { A.1.1 } 2 \pi-s \text {-vertex } & 10\end{array}$

$\begin{array}{lll}\text { A.1.2 } 4 \pi \text { and } 4 \pi-s \text { vertices } & 10\end{array}$

$\begin{array}{lll}\text { A.1.3 Counter terms } & 11\end{array}$

B Results for the octet and singlet form factors $\quad 12$

$\begin{array}{ll}\text { C Kinematical functions } & 13\end{array}$

\section{Introduction}

At first sight the scalar form factor of the pion appears to be a purely academic quantity since despite being a well-defined object in QCD, nature does not seem to provide a lowenergy scalar probe. The experimental information on the scalar $I=0 \pi \pi$ phase shift however serves as an input parameter in the dispersive representation of the form factor. Indirectly a comparison with experiment is therefore in principle possible.

Lattice QCD is the tool of choice for determining low energy properties of hadrons [1]. However, while computing the scalar form factor is in principle a straight forward procedure it turns out to be numerically much more complicated than the computation of the closely related pion vector form factor in the iso-spin limit [2-5]. The reason lies in the contribution of quark-disconnected Wick contractions to its defining correlation functions in the scalar case. There is no conceptual difficulty in treating these [5] but the computational effort is immense compared to quark-connected contributions. The disconnected part is therefore often neglected, not always providing supporting arguments that the systematic effect introduced in this way is under control. 
This paper provides the expressions for the contributions of the quark connected and the quark disconnected Wick contractions to the scalar form factor of the pion in chiral perturbation theory at next-to-leading order (NLO) [6-8]. As one might have expected and as first numerical studies indicate [5], the relative contribution of the quark-disconnected part to the form factor in the space-like region turns out to be small for small values of the momentum transfer. However, its contribution to the form factor above threshold and to the scalar radius which is defined as the form factor slope at vanishing momentum transfer turn out to be of about the same magnitude as the one of the quark-connected contribution.

While underlining the potential shortcomings of neglecting quark-disconnected contributions in lattice computations of QCD observables the results derived in this work allow to predict the disconnected contribution at NLO in the effective theory. Moreover, the arguments presented here justify the use of (partially) twisted boundary conditions in a lattice computation [9-14] allowing to compute the form factor on the finite lattice volume for arbitrary values of the momentum transfer.

The technique used to derive these predictions is based on ideas of $[15,16]$ which were formalised and applied to the two-point correlator of vector currents in QCD in [16]. After a brief summary of the idea the important steps in the computation for the scalar form factor within this frame work are presented for the theory with both $N_{f}=2$ and $N_{f}=2+1$ flavours, followed by an illustration and discussion of the results.

\section{Quark disconnected diagrams in chiral effective theory}

The technique will be briefly discussed for the example of the 2-flavour theory. The generalisation to the 2+1-flavour theory is straight forward and will become clear in the following sections. The scalar form factor of the pion is defined through

$$
\left\langle\pi^{i}\left(p^{\prime}\right)|\bar{u} u+\bar{d} d| \pi^{j}(p)\right\rangle=\delta^{i j} F_{S, 2}(t) .
$$

where $t=\left(p^{\prime}-p\right)^{2}$ is the squared momentum transfer and where the sub-script 2 indicates $N_{f}=2$ flavours. The matrix element on the l.h.s. is the ground state contribution to the Fourier transform of the QCD quark three point function

$$
\left\langle\mathcal{O}^{i}(z) S(y) \mathcal{O}^{i \dagger}(x)\right\rangle,
$$

constructed of the interpolating operators $O^{i}(x)=\bar{\psi}_{2} \tau^{i} \gamma_{5} \psi_{2}(x)$ and $S(x)=\bar{\psi}_{2}(x) \psi_{2}(x)$. The $\psi_{2}^{T}=(u, d)$ are $\mathrm{SU}(2)$ flavour vectors of $u$ - and $d$-quarks and the matrices $\tau_{i}=\sigma_{i} / 2$ are proportional to the Pauli matrices. On the level of the quark fields there are two classes of Wick-contractions as illustrated in figure 1.

As detailed in [16], by introducing an additional valence quark, $v$, which is degenerate with the light dynamical flavours, each Wick contraction can be rewritten in terms of a single fermionic correlation function defined in an un-physical theory. The physical result is recovered by summing over the correlation functions in the un-physical, partially quenched, theory $[15,17,18]$. In the case we consider here, e.g.,

$$
\left\langle\bar{u} \gamma_{5} d \bar{d} d \bar{d} \gamma_{5} u\right\rangle=\left\langle\bar{u} \gamma_{5} v \bar{v} d \bar{d} \gamma_{5} u\right\rangle+\left\langle\bar{u} \gamma_{5} d \bar{v} v \bar{d} \gamma_{5} u\right\rangle
$$



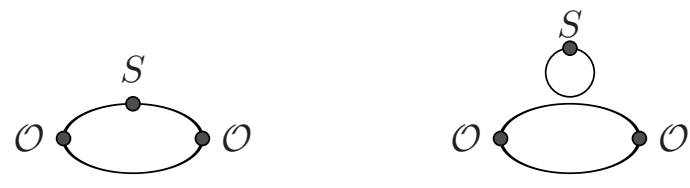

Figure 1. Wick contractions contributing to the scalar form factor.

An expression for the l.h.s. in the effective theory has been found many years ago at NLO $[6,8]$ and at NNLO in $[19,20]$ for both the $N_{f}=2$ and $N_{f}=2+1$ theory. This paper provides expressions for the ground state matrix elements of the terms on the r.h.s. of eq. (2.3) in partially quenched chiral perturbation theory (PQ $\chi \mathrm{PT})[15,17,18]$, which is an extension of chiral perturbation theory $[6,7]$ that provides an asymptotic low energy description of partially quenched QCD (PQQCD). Following [16], the flavour group is promoted to the graded $\mathrm{SU}(3 \mid 1)$ with the flavour vector $\psi_{3 \mid 1}^{T}=(u, d, v, g)$ extended by an additional valence and ghost quark. Both are assumed to be mass degenerate to the upand down quarks. The generators of the thus enlarged flavour group are $T^{a}, a=1, \ldots, 15$. We use the conventions also employed in $[16,21]$,

$$
T^{a}=\left(T^{a}\right)^{\dagger}, \quad \operatorname{Str}\left\{T^{a}\right\}=0, \quad \operatorname{Str}\left\{T^{a} T^{b}\right\}=\frac{1}{2} g^{a b}, \quad a=1, \ldots, 15,
$$

with the super-trace $\operatorname{Str}\{A\}=A_{11}+A_{22}+A_{33}-A_{44}$. The matrix $g^{a b}$ reads

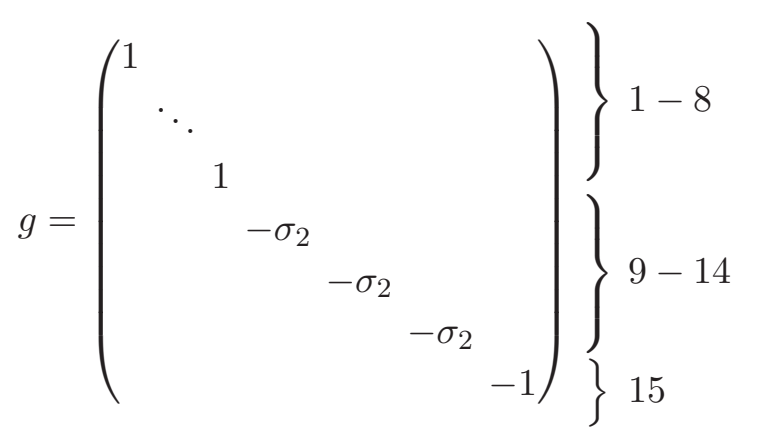

where $\sigma_{2}$ is the second Pauli matrix. $T^{1}, \ldots, T^{8}$ are the generators of the $\mathrm{SU}(3)$ subgroup that acts on the sea and valence components, $T^{9}, \ldots, T^{14}$ mix the quark with the ghost components, $T^{15}$ is the diagonal matrix $\operatorname{diag}(1,1,1,3) /(2 \sqrt{3})$. The matrix $T^{0}$ with $\operatorname{Str}\left\{T^{0}\right\}=1 / \sqrt{2}$ is proportional to the unit matrix.

In this theory the matrix element in eq. (2.1) can be written as

$$
\left\langle\pi^{1}\left|S^{0}+\frac{S^{8}-S^{15}}{\sqrt{3}}\right| \pi^{1}\right\rangle=\left\langle\pi^{1}\left|2 S^{4}\right| \pi^{1}\right\rangle+\left\langle\pi^{1}\left|S^{0}-\frac{2 S^{8}+S^{15}}{\sqrt{3}}\right| \pi^{6}\right\rangle,
$$

where the scalar source now is of the form $S^{i}=\bar{\psi}_{3 \mid 1} T^{i} \psi_{3 \mid 1}$.

All previous steps can also be applied to the 2+1-flavour theory. There, the SU(3) flavour symmetry is promoted to a graded $\mathrm{SU}(4 \mid 1)$ and the flavour content of the theory is 


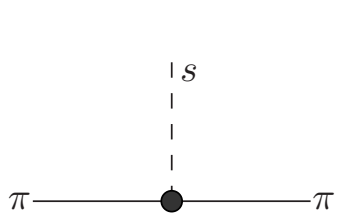

(a) tree level

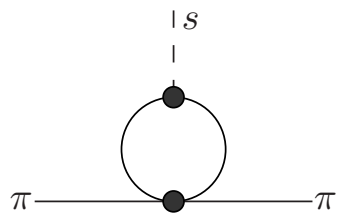

(b) bubble
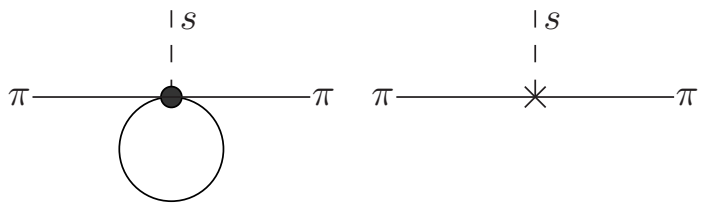

(c) tadpole

(d) counter

Figure 2. Contributing diagrams in the effective theory. Solid lines represent pions built of fermionic or bosonic quarks and the dashed line represents the external scalar source.

represented by the flavour vector $\psi_{4 \mid 1}^{T}=(u, d, s, v, g)$ with $s$ being the strange quark field. The graded group SU(4|1) has 24 generators and the super-trace as well as the metric $g^{a b}$ and $T^{0}$ introduced above for $\mathrm{SU}(3 \mid 1)$ need to be modified correspondingly.

In the next section the chiral effective theory corresponding to the $\mathrm{SU}(3 \mid 1)$ and $\mathrm{SU}(4 \mid 1)$ flavour symmetry groups is set up.

\section{$3 \quad \mathrm{PQ} \chi \mathrm{PT}$ for the scalar pion form factor}

The degrees of freedom of the partially quenched theory are parameterised in terms of $U=\exp \left(2 i \frac{\phi^{a} T^{a}}{F}\right)$, where the $\phi^{a}$ are the Goldstone-boson/fermion fields $(a=1, \ldots, 15$ for $\mathrm{SU}(3 \mid 1)$ and $a=1, \ldots, 24$ for $\mathrm{SU}(4 \mid 1))$. All vertices relevant for the computation at NLO of the scalar form factor (illustrated in figure $2 \mathrm{a}-2 \mathrm{c}$ and explicitly given in appendix A.1) can be derived from the leading order chiral Lagrangian $[6,7,15,17,18]$

$$
\mathcal{L}^{(2)}=\frac{F^{2}}{4} \operatorname{Str}\left\{\partial_{\mu} U \partial^{\mu} U^{\dagger}\right\}+\frac{F^{2}}{4} \operatorname{Str}\left\{\chi U^{\dagger}+U \chi^{\dagger}\right\}
$$

where $\chi=2 B(s+M)$. The mass matrix has the form

$$
\begin{array}{ll}
\mathrm{SU}(3 \mid 1) & \mathrm{SU}(4 \mid 1) \\
M=\operatorname{diag}\left(m_{q}, m_{q}, m_{q}, m_{q}\right), & M=\operatorname{diag}\left(m_{q}, m_{q}, m_{s}, m_{q}, m_{q}\right) .
\end{array}
$$

and we define the external scalar source as $s=2 T^{a} s^{a}$. The relevant counter terms illustrated in figure $2 \mathrm{~d}$ can be derived from the Lagrangian ,

$$
\begin{aligned}
\mathcal{L}^{(4)}= & L_{4} \operatorname{Str}\left\{\partial_{\mu} U\left(\partial^{\mu} U\right)^{\dagger}\right\} \operatorname{Str}\left\{\chi U^{\dagger}+U \chi^{\dagger}\right\}+L_{5} \operatorname{Str}\left\{\left(\partial_{\mu} U\left(\partial^{\mu} U\right)^{\dagger}\right)\left(\chi U^{\dagger}+U \chi^{\dagger}\right)\right\} \\
& +L_{6} \operatorname{Str}\left\{\chi U^{\dagger}+U \chi^{\dagger}\right\}^{2}+L_{8} \operatorname{Str}\left\{U \chi^{\dagger} U \chi^{\dagger}+\chi U^{\dagger} \chi U^{\dagger}\right\}^{2} .
\end{aligned}
$$

The partially quenched propagators are constructed in analogy to the procedure presented in detail in $[16,21]$. Note however that in the current paper we use Minkowski-signature. 


\section{Results for the form factor}

\subsection{Expressions in the effective theory at NLO}

For the 2-flavour theory the expressions for the form factor are

$$
\begin{aligned}
& F_{S, 2}^{\mathrm{F}}(t)=2 B\left\{1+\frac{1}{F^{2}}\left(-\frac{1}{2} \bar{A}\left(m_{\pi}^{2}\right)+\Lambda_{2}^{\mathrm{F}}+\frac{1}{2}\left(2 t-m_{\pi}^{2}\right) \bar{B}\left(m_{\pi}^{2}, t\right)\right)\right\}, \\
& F_{S, 2}^{\mathrm{C}}(t)=2 B\left\{1+\frac{1}{F^{2}}\left(-\frac{1}{2} \bar{A}\left(m_{\pi}^{2}\right)+\Lambda_{2}^{\mathrm{C}}+\frac{1}{2}\left(t-2 m_{\pi}^{2}\right) \bar{B}\left(m_{\pi}^{2}, t\right)\right)\right\}, \\
& F_{S, 2}^{\mathrm{D}}(t)=2 B\left\{0+\frac{1}{F^{2}}\left(\quad \Lambda_{2}^{\mathrm{D}}+\frac{1}{2}\left(t+m_{\pi}^{2}\right) \bar{B}\left(m_{\pi}^{2}, t\right)\right)\right\},
\end{aligned}
$$

where,

$$
\begin{aligned}
& \Lambda_{2}^{\mathrm{F}}=4\left\{m_{\pi}^{2}\left(-8 \tilde{L}_{4}^{r}-4 \tilde{L}_{5}^{r}+16 \tilde{L}_{6}^{r}+8 \tilde{L}_{8}^{r}\right)+t\left(2 \tilde{L}_{4}^{r}+\tilde{L}_{5}^{r}\right)\right\}, \\
& \Lambda_{2}^{\mathrm{C}}=4\left\{m_{\pi}^{2}\left(-4 \tilde{L}_{4}^{r}-4 \tilde{L}_{5}^{r}+8 \tilde{L}_{6}^{r}+8 \tilde{L}_{8}^{r}\right)+t \quad \tilde{L}_{5}^{r}\right\}, \\
& \Lambda_{2}^{\mathrm{D}}=4\left\{m_{\pi}^{2}\left(-4 \tilde{L}_{4}^{r} \quad+8 \tilde{L}_{6}^{r}\right) \quad+t 2 \tilde{L}_{4}^{r} \quad\right\} .
\end{aligned}
$$

and where $t=\left(p^{\prime}-p\right)^{2}$ is the squared momentum transfer between the two pions. The kinematical functions $\bar{A}\left(m^{2}\right)$ and $\bar{B}\left(m^{2}, t\right)$ are defined in appendix C. The superscripts $\mathrm{F}$, $\mathrm{C}$ and D indicate the Full form factor and the Connected and Disconnected contributions, respectively and the superscript $r$ indicates the subtraction of divergences in the $\overline{M S}$ scheme at subtraction scale $\mu=0.77 \mathrm{GeV}$. A tilde was added to the low-energy constants in order to distinguish them from the 3 -flavour ones. With

$$
\mathcal{A}=\frac{1}{6} \bar{A}\left(m_{\eta}^{2}\right)-\frac{1}{2} \bar{A}\left(m_{\pi}^{2}\right),
$$

the results for the $N_{f}=2+1$ theory are

$$
\begin{aligned}
F_{S, 3}^{\mathrm{F}}(t) & =2 B\left\{1+\frac{1}{F^{2}}\left(\mathcal{A}+\Lambda_{3}^{\mathrm{F}}\right.\right. \\
+ & \left.\left.\frac{m_{\pi}^{2}}{18} \bar{B}\left(m_{\eta}^{2}, t\right)+\frac{1}{2}\left(2 t-m_{\pi}^{2}\right) \bar{B}\left(m_{\pi}^{2}, t\right)+\frac{t}{4} \bar{B}\left(m_{K}^{2}, t\right) \quad\right)\right\}, \\
F_{S, 3}^{\mathrm{C}}(t)=2 B\left\{1+\frac{1}{F^{2}}\left(\mathcal{A}+\Lambda_{3}^{\mathrm{C}}\right.\right. & \left.\left.\quad+\frac{1}{2}\left(t-2 m_{\pi}^{2}\right) \bar{B}\left(m_{\pi}^{2}, t\right)+\frac{t}{4} \bar{B}\left(m_{K}^{2}, t\right)+\frac{m_{\pi}^{2}}{3} \bar{B}\left(m_{\eta}^{2}, m_{\pi}^{2}, t\right)\right)\right\}, \\
F_{S, 3}^{\mathrm{D}}(t)=2 B\left\{0+\frac{1}{F^{2}}\left(+\Lambda_{3}^{\mathrm{D}}\right.\right. & \left.\left.-\frac{m_{\pi}^{2}}{3} \bar{B}\left(m_{\eta}^{2}, m_{\pi}^{2}, t\right)\right)\right\}, \\
+ & \frac{m_{\pi}^{2}}{18} \bar{B}\left(m_{\eta}^{2}, t\right)+\frac{1}{2}\left(t+m_{\pi}^{2}\right) \bar{B}\left(m_{\pi}^{2}, t\right)
\end{aligned}
$$

where

$$
\begin{aligned}
& \Lambda_{3}^{\mathrm{F}}=4\left\{m_{\pi}^{2}\left(-6 L_{4}^{r}-4 L_{5}^{r}+12 L_{6}^{r}+8 L_{8}^{r}\right)+m_{K}^{2}\left(-4 L_{4}^{r}+8 L_{6}^{r}\right)+t\left(2 L_{4}^{r}+L_{5}^{r}\right)\right\}, \\
& \Lambda_{3}^{\mathrm{C}}=4\left\{m_{\pi}^{2}\left(-2 L_{4}^{r}-4 L_{5}^{r}+4 L_{6}^{r}+8 L_{8}^{r}\right)+m_{K}^{2}\left(-4 L_{4}^{r}+8 L_{6}^{r}\right)+t \quad L_{5}^{r}\right\} \text {, } \\
& \Lambda_{3}^{\mathrm{D}}=4\left\{m_{\pi}^{2}\left(-4 L_{4}^{r} \quad+8 L_{6}^{r}\right) \quad+t 2 L_{4}^{r}\right\} .
\end{aligned}
$$

The results for the octet and singlet scalar form factors

$$
\begin{aligned}
\left\langle\pi^{i}|\bar{u} u+\bar{d} d-2 \bar{s} s| \pi^{k}\right\rangle & =\delta^{i k} F_{S}^{8}(t), \\
\left\langle\pi^{i}|\bar{u} u+\bar{d} d+\bar{s} s| \pi^{k}\right\rangle & =\delta^{i k} F_{S}^{0}(t),
\end{aligned}
$$


are given in appendix B. An interesting observation is the absence of low-energy constants in the expression for the disconnected contribution to the octet form factor due to the vanishing super-trace of the octet-current. This allows for its parameter-free prediction at this order of the chiral expansion.

\subsection{Numerical results}

The chiral Lagrangian in the 2-flavour theory in [6] was presented in a different parameterisation which is related to the one used here through the relations $l_{3}=4\left(-2 \tilde{L}_{4}-\tilde{L}_{5}+\right.$ $\left.4 \tilde{L}_{6}+2 \tilde{L}_{8}\right)$ and $l_{4}=4\left(2 \tilde{L}_{4}+\tilde{L}_{5}\right)$, hence,

$$
\begin{aligned}
& \Lambda_{2}^{\mathrm{F}}=t l_{4}^{r} \quad+4 m_{\pi}^{2} l_{3}^{r}, \\
& \Lambda_{2}^{\mathrm{C}}=t\left(l_{4}^{r}-8 \tilde{L}_{4}^{r}\right)+4 m_{\pi}^{2}\left(l_{3}^{r}+4 \tilde{L}_{4}^{r}-8 \tilde{L}_{6}^{r}\right), \\
& \Lambda_{2}^{\mathrm{D}}=t\left(+8 \tilde{L}_{4}^{r}\right)+4 m_{\pi}^{2}\left(\quad-4 \tilde{L}_{4}^{r}+8 \tilde{L}_{6}^{r}\right) .
\end{aligned}
$$

The results for $F_{S, 2}^{\mathrm{F}}$ and $F_{S, 3}^{\mathrm{F}}$ agree with the ones found in [6, 8]. The low-energy parameters $\tilde{L}_{4}^{r}$ and $\tilde{L}_{6}^{r}$ entering $F_{S, 2}^{\mathrm{C}}$ and $F_{S, 2}^{\mathrm{D}}$ are in principle unknown from the 2-flavour theory - a corollary of the unphysical nature of the connected and the disconnected contributions considered on their own. The corresponding terms are naively present in the $\mathrm{SU}(2)$ chiral Lagrangian but trace-identities and the use of the equations of motion allow to reduce the relevant set of terms, hence the above linear combinations defining $l_{3}$ and $l_{4}$ in terms of the $\tilde{L}_{i}$.

In both the 2- and the 3-flavour theory the disconnected contribution is suppressed by power counting relative to the connected contribution and the full form factor, respectively. Power counting also suggests that the contribution of the disconnected contractions to the scalar radius $\left\langle r^{2}\right\rangle$ defined through

$$
F_{S}(t)=F_{S}(0)\left(1+\frac{1}{6}\left\langle r^{2}\right\rangle t+O\left(t^{2}\right)\right)
$$

(and similarly for the octet and singlet charge radius) can be of about the same magnitude. Since the tree-level contribution is purely real, the connected and the disconnected contractions also contribute democratically to the imaginary part of the form factor above the two-pion threshold in the time-like region.

In order to analyse the two contributions to the form factor more quantitatively we use the lattice estimates for the 3-flavour low-energy constants $L_{4}^{r}, L_{5}^{r}, L_{6}^{r}$ and $L_{8}^{r}$ by the RBC/UKQCD lattice collaboration $[22]^{1}$ as given in table 1. Further input parameters are $F=93 \mathrm{MeV}, m_{\pi}=0.135 \mathrm{MeV}, m_{K}=495 \mathrm{MeV}$ and $m_{\eta}=548 \mathrm{MeV}$. With this external input it is straight forward to analyse the 3-flavour case. Although $\tilde{L}_{4}^{r}$ and $\tilde{L}_{6}^{r}$ are not known they can be related to the low-energy constants $L_{4}^{r}$ and $L_{6}^{r}$ by matching the two

\footnotetext{
${ }^{1}$ See [1] for a summary of determinations of low-energy parameters in lattice QCD.
} 


\begin{tabular}{|ccc|}
\hline$L_{4}^{r}$ & {$[1,22]$} & $0.14 \times 10^{-3}$ \\
$L_{5}^{r}$ & {$[1,22]$} & $0.87 \times 10^{-3}$ \\
$L_{6}^{r}$ & {$[1,22]$} & $0.07 \times 10^{-3}$ \\
$L_{8}^{r}$ & {$[1,22]$} & $0.56 \times 10^{-3}$ \\
\hline
\end{tabular}

Table 1. Values for the 3-flavour low energy constants used in the illustration of the results. The subtraction scale is $\mu=0.77 \mathrm{GeV}$.

theories. In particular, we use

$$
\begin{array}{ll}
l_{3}^{r} & =4\left(-8 L_{4}^{r}-4 L_{5}^{r}+16 L_{6}^{r}+8 L_{8}^{r}\right)-\frac{1}{18} \tilde{L}\left(m_{\eta}^{2}\right), \\
l_{4}^{r} & =8 L_{4}^{r}+4 L_{5}^{r}-\frac{1}{4} \tilde{L}\left(m_{K}^{2}\right), \\
4 \tilde{L}_{4}^{r}-\tilde{8} L_{6}^{r} & =4 L_{4}^{r}-8 L_{6}^{r}-\frac{5}{4} \frac{1}{18} \tilde{L}\left(m_{\eta}^{2}\right)-\frac{1}{12} \frac{1}{N}, \\
\tilde{L}_{4}^{r} & =L_{4}^{r},
\end{array}
$$

where $\tilde{L}\left(m^{2}\right)=\frac{1}{16 \pi^{2}}\left(1+\ln \left(m^{2} / \mu^{2}\right)\right)$.

The dependence on the momentum of the full, the connected and the disconnected form factor are illustrated in the plots in figure 3. As anticipated $F_{S}^{\mathrm{D}}$ (dashed) is suppressed relative to $F_{S}^{\mathrm{C}}$ (dotted), predominantly due to the absence of a tree-level contribution in the former. Above threshold the contribution from the imaginary part of the form factor sets in and the quark disconnected contribution is larger than $50 \%$ over the whole range plotted. Figure 4 shows the scalar radius and as expected the disconnected contribution is not suppressed. In fact, both the connected and the disconnected contractions contribute to roughly equal parts.

The observations made here for the form factor $F_{S}$ apply qualitatively in the same way for the form factors $F_{S}^{0}$ and $F_{S}^{8}$.

\section{Applications}

Clearly this study has been motivated by the technical problems encountered in the computation in lattice QCD of observables receiving contributions from quark-disconnected diagrams. Here we highlight three ways in which the results of this paper might be of use:

- When computing the scalar form factor in lattice QCD with the aim of determining low-energy parameters it is possible to gain valuable information by comparing the dependence of the lattice data for only the connected part $F_{S}^{\mathrm{C}}$ on the quark mass and the momentum transfer, to the corresponding expressions in the effective theory for $N_{f}=2$ (eq. (4.1)) and $N_{f}=2+1$ (eq. (4.4)). A special case in this context is the octet scalar form factor (cf. appendix B) since the expression for the disconnected contribution is entirely parameter-free. 

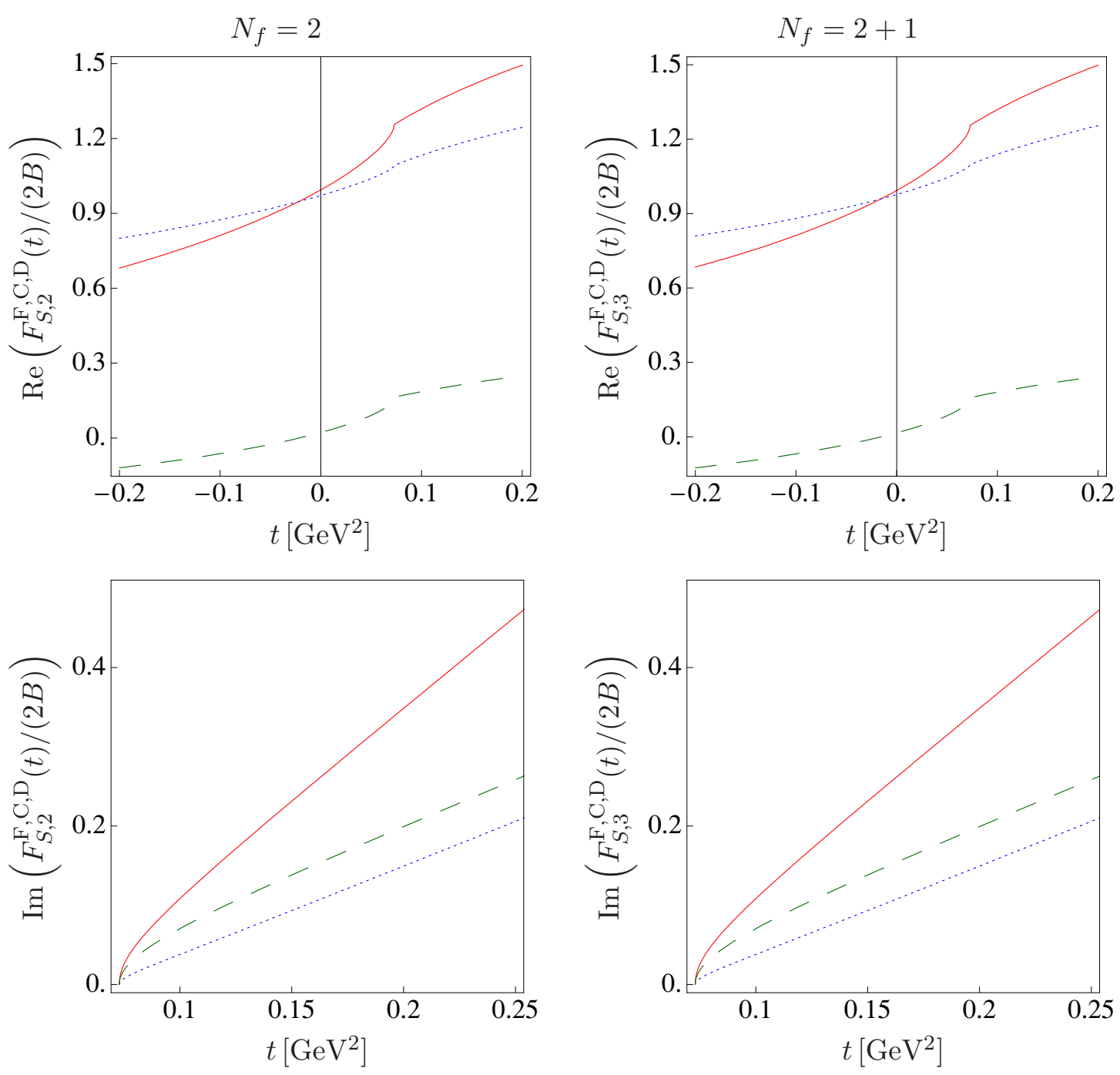

Figure 3. The plots show the momentum dependence of the real (top) and imaginary (bottom) parts of the scalar form factor for $N_{f}=2$ (left) and $N_{f}=2+1$ (right): Full form factor (solid red), connected contribution (dotted blue) and disconnected contribution (dashed green).

- If one is interested in the result for the full form factor $F_{S}^{\mathrm{F}}$, the only approach which will eventually provide a reliable control of systematic effects is a complete numerical evaluation in lattice QCD. An approximate picture for the form factor can be obtained by combining an evaluation of the connected contribution in lattice QCD with predictions for the disconnected contribution by chiral perturbation theory. With the exception of the octet form factor the latter of course relies on external input in terms of estimates of the low-energy constants.

- It was shown in [9-14] that by applying the quark boundary condition $q_{i}\left(x+\hat{L}_{k}\right)=$ $e^{i \theta_{k, i}} q_{i}(x)(i=u, d)$ the dispersion relation of a pseudo-scalar meson of mass $m$ consisting of the valence quark-fields $q_{u}$ and $q_{d}$ in the finite lattice volume of spatial extent 

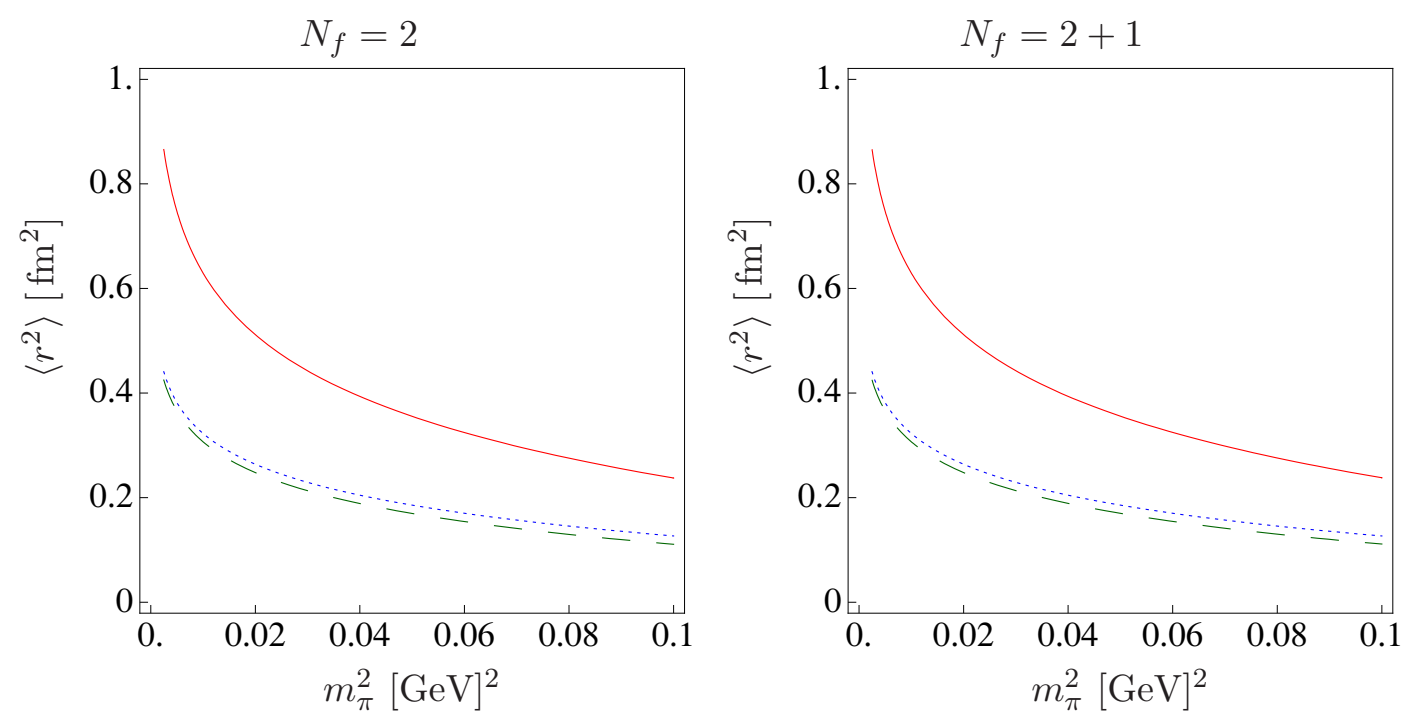

Figure 4. The plots show the scalar radius for $N_{f}=2$ (left) and $N_{f}=2+1$ (right) as a function of the squared pion mass $\left(m_{K}\right.$ is fixed to the physical value and $\left.m_{\eta}^{2}=\frac{4}{3} m_{K}^{2}-\frac{1}{3} m_{\pi}^{2}\right)$ : full radius (solid red), connected contribution (dotted blue) and disconnected contribution (dashed green).

$L$ takes the form $E\left(\theta_{u}, \theta_{d}\right)=\sqrt{m^{2}+\left(\theta_{u} / L-\theta_{d} / L\right)^{2}}$. While in this way allowing to tune the kinematics (partial) twisting only introduces exponentially suppressed and predictable finite volume effects for matrix elements with maximally one hadron in the initial or final state. In [23] the technique was then extended to meson 3pt functions, in particular the pion vector form factor and the $K \rightarrow \pi$ semi-leptonic form factors. The technique does however not work for the pion's scalar form factor since the effect of a twist applied to the $u$ - and/or $d$-quarks on the 1.h.s. of eq. (2.3) vanishes due to the flavour-diagonal nature of the scalar current. The connected term $\left\langle\bar{u} \gamma_{5} v \bar{v} d \bar{d} \gamma_{5} u\right\rangle$ however can be computed using this technique and the momentum transfer induced by partial twisting of the $u, d$ and $v$ quark is (assuming projection onto vanishing Fourier momenta)

$$
q^{2}=\left\{\left(E_{\pi}\left(\theta_{u}, \theta_{d}\right)-E\left(\theta_{u}, \theta_{v}\right)\right)^{2}-\left(\theta_{d} / L-\theta_{v} / L\right)^{2}\right\} .
$$

This technique allows to compute the connected part of the scalar form factor for any desired value of the momentum transfer and will be helpful in implementing the computational strategies suggested in the first two items.

\section{Conclusions and outlook}

This paper provides the expressions for quark-connected and quark-disconnected Wick contractions, respectively, contributing to the pion's scalar form factor and its radius in next-to-leading order (partially quenched) chiral perturbation theory. While the quark-disconnected part in the scalar form factor is sub-dominant, it turns out to contribute with about the same magnitude to the scalar radius as the quark-connected one. 
This result has implications for lattice QCD simulations where quark-disconnected contributions are often neglected ad hoc because of their typically bad signal-to-noise ratio. Computing quark-disconnected contributions in the effective theory can provide power-counting arguments for or against neglecting them for QCD-observables where chiral perturbation theory is expected to be a reliable effective description of the low-energy properties of the underlying fundamental theory. The example of the octet scalar form factor shows that for a suitable choice of the observable a prediction of the disconnected contribution in the effective theory is possible without loss of predictivity.

Quark-disconnected contractions contribute to a large class of matrix elements relevant for the precision phenomenology of the Standard Model. Examples are the yet to be fully understood process $K \rightarrow \pi \pi$ or iso-spin breaking effects. The technique employed here and previously in [16] can potentially be applied to guide the computation of quarkdisconnected contributions in these cases.

\section{Acknowledgments}

The author would like to thank Martin Lüscher for comments on the manuscript and Michele Della Morte for fruitful discussions.

\section{A Vertices in the partially quenched theory}

The Feynman rules in partially quenched chiral perturbation theory differ from the ones in the purely bosonic theory since the commutators of the involved generators depend on their grading $\eta^{a}$, i.e. $\eta^{a}=0(1)$ if $T^{a}$ generates a boson(fermion). In the following we provide expression for the relevant vertices that derive from the Lagrangians (3.1) and (3.3).

\section{A.1 Vertices}

\section{A.1.1 $2 \pi-s$-vertex}

The vertex illustrated in figure $5 \mathrm{a}$ is

$$
\begin{aligned}
V_{2 \pi s}^{\gamma, \alpha, \beta}=-i 2 B\left\{(-1)^{\eta^{\alpha}\left(\eta^{\beta}+\eta^{\gamma}\right)}\right. & \left(\operatorname{Str}\left\{T^{\alpha} T^{\gamma} T^{\beta}\right\}+(-1)^{\eta^{\beta} \eta^{\gamma}} \operatorname{Str}\left\{T^{\alpha} T^{\beta} T^{\gamma}\right\}\right. \\
+ & \left.\left.\operatorname{Str}\left\{T^{\gamma} T^{\beta} T^{\alpha}\right\}+(-1)^{\eta^{\beta} \eta^{\gamma}} \operatorname{Str}\left\{T^{\beta} T^{\gamma} T^{\alpha}\right\}\right)\right\}
\end{aligned}
$$

\section{A.1.2 $4 \pi$ and $4 \pi-s$ vertices}

The $4 \pi-s$ vertex in figure $5 \mathrm{~b}$ is

$$
\begin{aligned}
V_{4 \pi s}^{\gamma, \delta, \epsilon, \alpha, \beta}=i \frac{2}{3} \frac{B}{F^{2}} & \sum_{\mathcal{P}} \mathcal{S}(\sigma(\alpha), \sigma(\beta), \sigma(\gamma), \sigma(\delta)) \\
\times\left\{(-1)^{\epsilon}\left(\eta^{\alpha}+\eta^{\beta}+\eta^{\gamma}+\eta^{\delta}\right)\right. & \operatorname{Str}\left\{T^{\epsilon} T^{\sigma(\alpha)} T^{\sigma(\beta)} T^{\sigma(\gamma)} T^{\sigma(\delta)}\right\} \\
+ & \left.\operatorname{Str}\left\{T^{\sigma(\alpha)} T^{\sigma(\beta)} T^{\sigma(\gamma)} T^{\sigma(\delta)} T^{\epsilon}\right\}\right\},
\end{aligned}
$$




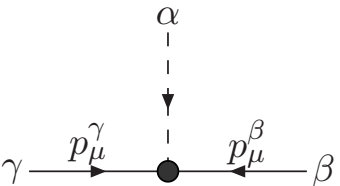

(a) $V_{2 \pi}^{\gamma, \alpha, \beta}$

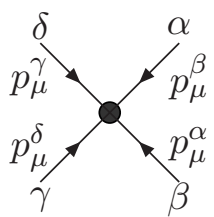

(c) $V_{4 \pi, \text { kinetic }}^{\alpha, \beta, \gamma, \delta}, V_{4 \pi, \text { mass }}^{\alpha, \beta, \gamma, \delta}$

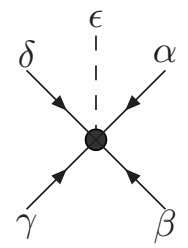

(b) $V_{4 \pi-s}^{\alpha, \beta, \gamma, \delta, \epsilon}$

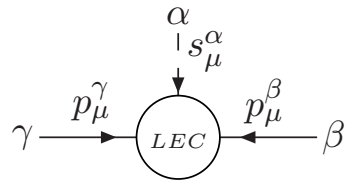

(d) $V_{l_{3,4}}^{\alpha, \beta, \gamma}$

Figure 5. Vertices entering the computation of the scalar form factor.

and the vertex deriving from the mass term as in figure $5 \mathrm{c}$ is

$$
V_{4 \pi M}^{\gamma, \delta, \alpha, \beta}=i \frac{2}{3} \frac{B}{F^{2}} \sum_{\mathcal{P}} \mathcal{S}(\sigma(\alpha), \sigma(\beta), \sigma(\gamma), \sigma(\delta)) \operatorname{Str}\left\{M T^{\sigma(\alpha)} T^{\sigma(\beta)} T^{\sigma(\gamma)} T^{\sigma(\delta)}\right\}
$$

Here $\mathcal{P}$ indicates that the sum is over all permutations $\sigma$ of the indices. Starting from $(\alpha, \beta, \gamma, \delta)$ a given permutation $(\sigma(\alpha), \sigma(\beta), \sigma(\gamma), \sigma(\delta))$ may be reached by a series of exchanges of pairs of neighbouring indices. $\mathcal{S}(\alpha, \beta, \gamma, \delta)$ is the product of signs that are picked up in each such exchange of two indices depending on its grading. For example, for $(\sigma(\alpha), \sigma(\beta), \sigma(\gamma), \sigma(\delta))=(\gamma, \beta, \alpha, \delta), S(\gamma, \beta, \alpha, \delta)=(-1)^{\eta^{\gamma}\left(\eta^{\beta}+\eta^{\alpha}\right)+\eta^{\beta} \eta^{\alpha}}$.

The four point vertex deriving from the kinetic term is

$$
\begin{aligned}
V_{4 \pi, \text { kin }}^{\gamma, \delta, \beta}=i \frac{1}{F^{2}} \sum_{\mathcal{P}} \mathcal{S}(\sigma(\alpha) & , \sigma(\beta), \sigma(\gamma), \sigma(\delta)) P(\sigma(\alpha), \sigma(\beta), \sigma(\gamma), \sigma(\delta)) \\
\times & \operatorname{Str}\left\{T^{\sigma(\alpha)} T^{\sigma(\beta)} T^{\sigma(\gamma)} T^{\sigma(\delta)}\right\}
\end{aligned}
$$

where $P(a, b, c, d)=\frac{2}{3} p^{a} \cdot p^{b}-\frac{1}{3} p^{a} \cdot p^{c}+\frac{1}{3} p^{a} \cdot p^{d}-\frac{1}{3} p^{b} \cdot p^{d}-p^{b} \cdot p^{c}+\frac{2}{3} p^{c} \cdot p^{d}$. In the case where all external legs are bosons the known vertices of $\mathrm{SU}(N)$ chiral perturbation theory are recovered.

\section{A.1.3 Counter terms}

The counter terms needed derive from $\mathcal{L}^{(4)}$ as given in eq. (3.3) and the corresponding vertices with the indices associated to the external legs as illustrated in figure $5 \mathrm{~d}$ are (we 
assume here that the external legs fulfil $\eta^{\beta}=\eta^{\gamma}=0$ ),

$$
\begin{aligned}
& V_{L_{4}}^{\gamma, \alpha, \beta}=-i \frac{32 L_{4} B}{F^{2}} p \cdot p^{\prime} \operatorname{Str}\left\{T^{\alpha}\right\} \operatorname{Str}\left\{T^{\beta} T^{\gamma}+T^{\gamma} T^{\beta}\right\}, \\
& V_{L_{5}}^{\gamma, \alpha, \beta}=-i \frac{32 L_{5} B}{F^{2}} p \cdot p^{\prime} \operatorname{Str}\left\{T^{\alpha}\left(T^{\beta} T^{\gamma}+T^{\gamma} T^{\beta}\right)\right\}, \\
& V_{L_{6}}^{\gamma, \alpha, \beta}=+i \frac{128 L_{6} B}{F^{2}}\left\{\operatorname{Str}\{M\} \operatorname{Str}\left\{T^{\alpha}\left(T^{\beta} T^{\gamma}+T^{\gamma} T^{\beta}\right)\right\}\right. \\
& \left.+\operatorname{Str}\left\{T^{\alpha}\right\} \operatorname{Str}\left\{M\left(T^{\beta} T^{\gamma}+T^{\gamma} T^{\beta}\right)\right\}\right\}, \\
& V_{L_{8}}^{\gamma, \alpha, \beta}=-i \frac{64 L_{8} B}{F^{2}}\left\{\operatorname{Str}\left\{T^{\alpha} M T^{\beta} T^{\gamma}\right\}+\operatorname{Str}\left\{T^{\alpha} M T^{\gamma} T^{\beta}\right\}\right. \\
& +\left(\operatorname{Str}\left\{M T^{\alpha} T^{\beta} T^{\gamma}\right\}+\operatorname{Str}\left\{M T^{\alpha} T^{\gamma} T^{\beta}\right\}\right) \\
& \left.+2\left(\operatorname{Str}\left\{M T^{\beta} T^{\alpha} T^{\gamma}\right\}+\operatorname{Str}\left\{M T^{\gamma} T^{\alpha} T^{\beta}\right\}\right)\right\} .
\end{aligned}
$$

\section{B Results for the octet and singlet form factors}

Here we provide the results for the octet and the singlet scalar form factor as defined in eq. (4.6),

$$
\begin{aligned}
& F_{S, 3}^{\mathrm{F}, 8}(t)=2 B\left\{1+\frac{1}{F^{2}}\left(\mathcal{A}+\Lambda_{3}^{\mathrm{F}, 8}\right.\right. \\
& \left.\left.-\frac{m_{\pi}^{2}}{6} \bar{B}\left(m_{\eta}^{2}, t\right)+\frac{1}{2}\left(2 t-m_{\pi}^{2}\right) \bar{B}\left(m_{\pi}^{2}, t\right)-\frac{t}{4} \bar{B}\left(m_{K}^{2}, t\right) \quad\right)\right\}, \\
& F_{S, 3}^{\mathrm{C}, 8}(t)=2 B\left\{1+\frac{1}{F^{2}}\left(\mathcal{A}+\Lambda_{3}^{\mathrm{C}, 8}\right.\right. \\
& \left.+\frac{1}{2}\left(t-2 m_{\pi}^{2}\right) \bar{B}\left(m_{\pi}^{2}, t\right)+\frac{t}{4} \bar{B}\left(m_{K}^{2}, t\right)+\frac{m_{\pi}^{2}}{3} \bar{B}\left(m_{\eta}^{2}, m_{\pi}^{2}, t\right)\right\}, \\
& F_{S, 3}^{\mathrm{D}, 8}(t)=2 B\left\{\quad \frac { 1 } { F ^ { 2 } } \left(+\Lambda_{3}^{\mathrm{D}, 8}\right.\right. \\
& \left.-\frac{m_{\pi}^{2}}{6} \bar{B}\left(m_{\eta}^{2}, t\right)+\frac{1}{2}\left(t+m_{\pi}^{2}\right) \bar{B}\left(m_{\pi}^{2}, t\right)-\frac{t}{2} \bar{B}\left(m_{K}^{2}, t\right)-\frac{m_{\pi}^{2}}{3} \bar{B}\left(m_{\eta}^{2}, m_{\pi}^{2}, t\right)\right\},
\end{aligned}
$$

and

$$
\begin{aligned}
& F_{S, 3}^{\mathrm{F}, 0}(t)=2 B\left\{1+\frac{1}{F^{2}}\left(\mathcal{A}+\Lambda_{3}^{\mathrm{F}, 0}\right.\right. \\
& \left.+\frac{m_{\pi}^{2}}{6} \bar{B}\left(m_{\eta}^{2}, t\right)+\frac{1}{2}\left(2 t-m_{\pi}^{2}\right) \bar{B}\left(m_{\pi}^{2}, t\right)+\frac{t}{2} \bar{B}\left(m_{K}^{2}, t\right)\right\}, \\
& F_{S, 3}^{\mathrm{C}, 0}(t)=2 B\left\{1+\frac{1}{F^{2}}\left(\mathcal{A}+\Lambda_{3}^{\mathrm{C}, 0}\right.\right. \\
& \left.+\frac{1}{2}\left(t-2 m_{\pi}^{2}\right) \bar{B}\left(m_{\pi}^{2}, t\right)+\frac{t}{4} \bar{B}\left(m_{K}^{2}, t\right)+\frac{m_{\pi}^{2}}{3} \bar{B}\left(m_{\eta}^{2}, m_{\pi}^{2}, t\right)\right\}, \\
& F_{S, 3}^{\mathrm{D}, 0}(t)=2 B\left\{\quad \frac { 1 } { F ^ { 2 } } \left(+\Lambda_{3}^{\mathrm{D}, 1}\right.\right. \\
& \left.+\frac{m_{\pi}^{2}}{6} \bar{B}\left(m_{\eta}^{2}, t\right)+\frac{1}{2}\left(t+m_{\pi}^{2}\right) \bar{B}\left(m_{\pi}^{2}, t\right)+\frac{t}{4} \bar{B}\left(m_{K}^{2}, t\right)-\frac{m_{\pi}^{2}}{3} \bar{B}\left(m_{\eta}^{2}, m_{\pi}^{2}, t\right)\right\} .
\end{aligned}
$$

These form factors have the following dependence on low-energy constants:

$$
\begin{aligned}
& \Lambda_{3}^{\mathrm{F}, 8}=4\left\{m_{\pi}^{2}\left(-2 L_{4}^{r}-4 L_{5}^{r}+4 L_{6}^{r}+8 L_{8}^{r}\right)+m_{K}^{2}\left(-4 L_{4}^{r}+8 L_{6}^{r}\right)+t L_{5}^{r}\right\}, \\
& \Lambda_{3}^{\mathrm{C}, 8}=4\left\{m_{\pi}^{2}\left(-2 L_{4}^{r}-4 L_{5}^{r}+4 L_{6}^{r}+8 L_{8}^{r}\right)+m_{K}^{2}\left(-4 L_{4}^{r}+8 L_{6}^{r}\right)+t L_{5}^{r}\right\}, \\
& \Lambda_{3}^{\mathrm{D}, 8}=0
\end{aligned}
$$


and

$$
\begin{aligned}
& \Lambda_{3}^{\mathrm{F}, 0}=4\left\{m_{\pi}^{2}\left(-8 L_{4}^{r}-4 L_{5}^{r}+16 L_{6}^{r}+8 L_{8}^{r}\right)+m_{K}^{2}\left(-4 L_{4}^{r}+8 L_{6}^{r}\right)+t\left(3 L_{4}^{r}+L_{5}^{r}\right)\right\}, \\
& \Lambda_{3}^{\mathrm{C}, 0}=4\left\{m_{\pi}^{2}\left(-2 L_{4}^{r}-4 L_{5}^{r}+4 L_{6}^{r}+8 L_{8}^{r}\right)+m_{K}^{2}\left(-4 L_{4}^{r}+8 L_{6}^{r}\right)+t \quad L_{5}^{r}\right\}, \\
& \Lambda_{3}^{\mathrm{D}, 0}=4\left\{m_{\pi}^{2}\left(-6 L_{4}^{r}+12 L_{6}^{r}\right)+t 3 L_{4}^{r} \quad\right\} .
\end{aligned}
$$

Note that $F_{S}^{\mathrm{D}, 8}$ is an entirely parameter-independent prediction for the disconnected contribution.

\section{Kinematical functions}

Here we provide the expressions for the kinematical functions $\bar{A}\left(m^{2}\right)$ (tadpole), $\bar{B}\left(m^{2}, \tilde{m}^{2}, t\right)$ and $\bar{B}\left(m^{2}, t\right)$ (both unitary contributions)

$$
\begin{aligned}
\bar{A}\left(m^{2}\right)= & -\frac{m^{2}}{16 \pi^{2}} \ln \left(m^{2} / \mu^{2}\right), \\
\bar{B}\left(m^{2}, \tilde{m}^{2}, t\right)= & -\frac{1}{16 \pi^{2}} \frac{m^{2} \ln \left(m^{2} / \mu^{2}\right)-\tilde{m}^{2} \ln \left(\tilde{m}^{2} / \mu^{2}\right)}{m^{2}-\tilde{m}^{2}} \\
& +\frac{1}{32 \pi^{2}}\left(2+\left(-\frac{\Delta}{t}+\frac{\Sigma}{\Delta}\right) \ln \left(m^{2} / \tilde{m}^{2}\right)-\frac{\nu}{t} \ln \frac{(t+\nu)^{2}-\Delta^{2}}{(t-\nu)^{2}-\Delta^{2}}\right), \\
\bar{B}\left(m^{2}, t\right)= & \lim _{\tilde{m}^{2} \rightarrow m^{2}} \bar{B}\left(m^{2}, \tilde{m}^{2}, t\right),
\end{aligned}
$$

where, as in e.g. [7], $\Delta=m^{2}-\tilde{m}^{2}, \Sigma=m^{2}+\tilde{m}^{2}$ and $\left.\nu^{2}=\left(t-(m+\tilde{m})^{2}\right)\left(t-(m-\tilde{m})^{2}\right)\right)$.

Open Access. This article is distributed under the terms of the Creative Commons Attribution Noncommercial License which permits any noncommercial use, distribution, and reproduction in any medium, provided the original author(s) and source are credited.

\section{References}

[1] G. Colangelo et al., Review of lattice results concerning low energy particle physics, Eur. Phys. J. C 71 (2011) 1695 [arXiv: 1011.4408] [InSPIRE].

[2] Bern-Graz-Regensburg (BGR) collaboration, S. Capitani, C. Gattringer and C. Lang, A lattice calculation of the pion form-factor with Ginsparg-Wilson-type fermions, Phys. Rev. D 73 (2006) 034505 [hep-lat/0511040] [INSPIRE].

[3] P. Boyle et al., The pion's electromagnetic form-factor at small momentum transfer in full lattice QCD, JHEP 07 (2008) 112 [arXiv:0804.3971] [INSPIRE].

[4] ETM collaboration, R. Frezzotti, V. Lubicz and S. Simula, Electromagnetic form factor of the pion from twisted-mass lattice $Q C D$ at $N_{f}=2$, Phys. Rev. D 79 (2009) 074506 [arXiv: 0812.4042] [INSPIRE].

[5] JLQCD and TWQCD collaborations, S. Aoki et al., Pion form factors from two-flavor lattice QCD with exact chiral symmetry, Phys. Rev. D 80 (2009) 034508 [arXiv:0905.2465] [INSPIRE].

[6] J. Gasser and H. Leutwyler, Chiral perturbation theory to one loop, Annals Phys. 158 (1984) 142 [INSPIRE]. 
[7] J. Gasser and H. Leutwyler, Chiral perturbation theory: expansions in the mass of the strange quark, Nucl. Phys. B 250 (1985) 465 [InSPIRE].

[8] J. Gasser and H. Leutwyler, Low-energy expansion of meson form-factors, Nucl. Phys. B 250 (1985) 517 [inSPIRE].

[9] C. Sachrajda and G. Villadoro, Twisted boundary conditions in lattice simulations, Phys. Lett. B 609 (2005) 73 [hep-lat/0411033] [INSPIRE].

[10] P.F. Bedaque and J.-W. Chen, Twisted valence quarks and hadron interactions on the lattice, Phys. Lett. B 616 (2005) 208 [hep-lat/0412023] [INSPIRE].

[11] P.F. Bedaque, Aharonov-Bohm effect and nucleon nucleon phase shifts on the lattice, Phys. Lett. B 593 (2004) 82 [nucl-th/0402051] [InSPIRE].

[12] G. de Divitiis, R. Petronzio and N. Tantalo, On the discretization of physical momenta in lattice QCD, Phys. Lett. B 595 (2004) 408 [hep-lat/0405002] [INSPIRE].

[13] B.C. Tiburzi, Flavor twisted boundary conditions and the nucleon axial current, Phys. Lett. B 617 (2005) 40 [hep-lat/0504002] [INSPIRE].

[14] UKQCD collaboration, J. Flynn, A. Jüttner and C. Sachrajda, A numerical study of partially twisted boundary conditions, Phys. Lett. B 632 (2006) 313 [hep-lat/0506016] [INSPIRE].

[15] S.R. Sharpe and N. Shoresh, Physical results from unphysical simulations, Phys. Rev. D 62 (2000) 094503 [hep-lat/0006017] [INSPIRE].

[16] M. Della Morte and A. Jüttner, Quark disconnected diagrams in chiral perturbation theory, JHEP 11 (2010) 154 [arXiv:1009.3783] [INSPIRE].

[17] C.W. Bernard and M.F. Golterman, Chiral perturbation theory for the quenched approximation of QCD, Phys. Rev. D 46 (1992) 853 [hep-lat/9204007] [INSPIRE].

[18] C.W. Bernard and M.F. Golterman, Partially quenched gauge theories and an application to staggered fermions, Phys. Rev. D 49 (1994) 486 [hep-lat/9306005] [INSPIRE].

[19] J. Bijnens, G. Colangelo and P. Talavera, The vector and scalar form-factors of the pion to two loops, JHEP 05 (1998) 014 [hep-ph/9805389] [INSPIRE].

[20] J. Bijnens and P. Dhonte, Scalar form-factors in SU(3) chiral perturbation theory, JHEP 10 (2003) 061 [hep-ph/0307044] [INSPIRE].

[21] L. Giusti and M. Lüscher, Chiral symmetry breaking and the Banks-Casher relation in lattice QCD with Wilson quarks, JHEP 03 (2009) 013 [arXiv:0812.3638] [INSPIRE].

[22] RBC-UKQCD collaboration, C. Allton et al., Physical results from $2+1$ flavor domain wall $Q C D$ and $\mathrm{SU}(2)$ chiral perturbation theory, Phys. Rev. D 78 (2008) 114509 [arXiv: 0804.0473] [INSPIRE].

[23] P. Boyle, J. Flynn, A. Jüttner, C. Sachrajda and J. Zanotti, Hadronic form factors in lattice QCD at small and vanishing momentum transfer, JHEP 05 (2007) 016 [hep-lat/0703005] [INSPIRE]. 